\title{
The LQAC-4, last update of the Large Quasar Astrometric Catalogue
}

\section{J. Souchay ${ }^{1}$, A. H. Andrei ${ }^{2}$, C. Barache ${ }^{1}$, F. Taris ${ }^{1}$, C. Gattano ${ }^{1}$ and B. Coelho ${ }^{2}$}

${ }^{1}$ SYRTE, Observatoire de Paris

PSL research Univ., CNRS Sorbonne Univ. Paris 06, LNE

61, avenue de l'observatoire, 75014 Paris, France

email: Jean.Souchay@obspm.fr

${ }^{2}$ Observ. do Valongo, Universidade Federal do Rio de Janeiro

Ladeira Pedro Antonio 43, Saude, Rio de Janeiro-REJ, CEP 20080-90, Brazil

email: bcoelho@astro.ufrj.br

\begin{abstract}
Thanks to their point-like structure and to their lack of significant proper motion, quasars represent the ideal objects for modeling quasi-inertial directions in space. For that reason the present primary conventional reference frame, the ICRF-2, is constructed from the set of the celestial coordinates of a sample of extragalactic objects, whose the very large majority are quasars. Thus any newly discovered quasar must be considered as a potential future ideal astrometric marker. Therefore compiling all the recorded quasars at a given epoch looks as a useful task. This constitutes the aim of the LQAC (Large Quasar Astrometric Catalogue). We present here the contents of the future release of this catalogue quoted as the LQAC-4, insisting on the related strategy of compilation. Preliminary results concerning the cross-identification with the Gaia DR1 catalogue are emphasized
\end{abstract}

Keywords. quasars : general, catalogs, surveys

\section{Introduction}

Soon after the first quasar was discovered, in the early 60s, continuous efforts have been made to compile the whole set of known quasars with regular updates. As any other type of celestial object, the interest of gathering in a same catalogue all the quasars can be considered as a commendable effort for the astronomical and astrophysical community. First this kind of work constitutes a useful tool for any researcher wanting to know the positions of all the already recorded objects. Second it gives some information about some of the fundamental characteristics as the redshift, the apparent magnitudes at several wavelengths, the absolute magnitude computed from suitable algorithms etc... Third it serves as a basis for general studies as statistics with respect to the parameters above, as well to sky coverage. Fourth it can be used as a kind of reference catalogue to identify the quasars present in the recently published Gaia DR1 release.

For these reasons continuous efforts have been made for more than 25 years by the same authors (Véron-Cetty and Véron (1984), Véron-Cetty and Véron (2010)) to construct an all-sky compilation of all the quasars detected so far. Their last release in 2010, the 13 th one, contained 133336 quasars together with an additional set of 34231 active galaxies (AGNs).

Then such a compilation work was continued by a new group (Souchay et al. (2009)) in the form of what is named the Large Quasar Astrometric Catalogue (LQAC), containing 113666 objects. Two up-dated catalogues followed, the LQAC-2 (Souchay et al. (2012)) and the LQAC-3 (Souchay et al. (2015)) with respectively 187504 and 321957 objects. 
These considerable consecutive increases are essentially due to the inclusion of successive releases of the Sloan Digital Sky Survey (SDSS), by far the main contributor.

In the following we discuss the construction of the fourth up-date of the LQAC, named as LQAC-4, which is scheduled to publication in the very near future.

\section{The construction of the LQAC-4}

The principle of compilation of the LQAC-4 (Gattano et al. (2017)) is identical to the LQAC-3 (Souchay et al. (2015)). It is built in a large part from a compilation of relatively large catalogues of quasars, four of them in the radio VLBI domain, five of them in the optical one. In the following we present the set of these nine original large catalogues involved. They are ranged in the following in an a priori decreasing order of accuracy of determination of the celestial coordinates of the objects. An alphabetic flag, from A to I is given according to this order.

\section{Four radio VLBI catalogues}

The four following radio catalogues have been elaborated from Very Long Baseline Interferometry (VLBI) observations. They are characterized by a relatively small number of objects but a very good astrometric accuracy.

- The ICRF-2 (flag "A")

The International Celestial Reference Frame, 2nd. release, called the ICRF-2 (Ma et al. (2009), Fey et al. (2015)) is the primary reference frame for all astronomers. It contains the positions, i.e. the celestial coordinates of 3414 compact radio-sources (mainly quasars but also some AGNs and BLAC). These coordinates are given with a noise floor estimated at 40 mas. They implicitly define the axes of the frame with a stability of 10 pas. Consequently the ICRF-2 is by far the most accurate catalogue of extragalactic objects in terms of celestial coordinates.

- The RFC/ VLBA (flag "B")

The Radio Fundamental Catalog (RFC) comes from a VLBI Astrometric global solution using all the available VLBI observations carried out in the frame of various surveys in different bandwidths, mainly at $8.6 \mathrm{GHz}$ and $2.2 \mathrm{GHz}$. The leading observational program is the VCS (VLBA calibrator survey) consisting in several thousands of $24 \mathrm{~h}$ continuous VLBI sessions playing a leading role in the astrometric performance. The catalogue is regularly updated with more sources and more observations per source. For the LQAC-4 we used the RFC2013 release already considered in the LQAC-3 and containing 7213 objects instead of the 5198 objects present in the LQAC-2.

- The VLA Catalogue (flag "C")

The Very Large Array (VLA) interferometer consists of 27 radio antennas of $25 \mathrm{~m}$ diameter dispatched in a Y shape at St. Augustin (New Mexico, USA). This gives the same resolution as one antenna of $36 \mathrm{~km}$-across, with the sensitivity of a $130 \mathrm{~m}$ dish. The catalog used in the LQAC-4 is exactly the same as in the LQAC-3. It contains 1701 quasars with an astrometric accuracy of roughly 10mas. (Claussen (2006).

- The JVAS Catalogue (flag "D")

The Jodrell Bank VLA Astrometric Survey (JVAS) catalog (Browne et al. (2009), Wilkinson et al. (1998)) contains 2118 compact radiosources uniformly distributed in the northern hemisphere with a peak flux density $>50 \mathrm{mJy}$ at a resolution of 200 mas at 8.4 GHz. The rms accuracy of the position of sources ranges between 10 mas and 55mas 
Five optical catalogues

The five following big catalogues of quasars contain information at optical wavelenths. Notice that two of them, the FIRST and the Hewitt and Burbridge ones, include substantial additional information in the radio domain.

- The SDSS Catalogue (flag "E")

The Sloan Digital Sky Survey (SDSS) constitutes by far the largest catalogue of the LQAC-4 compilation. Thanks to its recent up-date, the DR12Q by Pâris et al. (2017), it gathers 384834 elements. That constitutes a big increase with respect to the 262535 SDSS quasars in the LQAC-3, and represents $86.7 \%$ of the total number of objects in the LQAC-4. Nevertheless it covers roughly only one quarter of the sky, staring from observations with a dedicated $2.5 \mathrm{~m}$ telescope at Apache Point, New Mexico (USA). Images are obtained in five broad optical bands, designated as $u, g, r, i, z$. Astrometric calibration yields an accuracy of 45 mas per coordinate when reduced using the USNO CCD Astrograph Catalogue (Pier et al., 2003.

- The 2QZ Catalogue (flag "F")

The 2-degree Field (2dF) quasar redshift survey, quoted as 2QZ (Croom et al., 2004) is the second largest contributor, with a number of 23660 objects. It is based on a preselection of quasar candidates starting from color criteria and a spectroscopic follow-up completed by a minimization technique fitting each spectrum to a number of stellar and extragalactic objects templates. The survey area includes 30 fields arranged in $75^{\circ} \times 5^{\circ}$ declination strips passing across the South and North Galactic caps.

- The 2SLAQ Catalogue (flag "G")

The 2SLAQ Survey, which stands for the 2dF-Sloan Digital Sky Survey luminous red galaxy (LRG) and QSO Survey (2dF-SDSS LRG and QSO) (da Angela et al. 2008), combines photometric and spectroscopic data respectively from the Sloan telescope and from the $2 \mathrm{dF}$. It is an extension of the $2 \mathrm{QZ}$ survey above, at fainter magnitudes, and includes a total of 9058 QSO's.

- The FIRST Catalogue (flag "H")

The FIRST catalogue used in the LQAC-4 consists in a combination of three successive releases matching the radio NRAO VLA survey (Becker et al., 2001) with an optical catalogue obtained by digitization of plates coming from the PSS (Palomar Sky Survey).

- Hewitt and Burbridge Catalogue (flag "H")

The HB catalogue (Hewitt and Burbridge, 1993) contains 6720 objects representing a majority of the quasars known up to the epoch it was completed, that is to say in 1992 .

One complementary catalogue of quasar (Véron-Cetty and Véron (2010)), flag "M"

For the LQAC-4 the VV2010 catalogue (Véron-Cetty and Véron (2010)) was used to extract the 14509 quasars not included in the compilation obtained from the nine quasar catalogues described above. That constitutes a mere $3.26 \%$ of the whole LQAC- 4 .

\section{Cross-identification with Gaia DR1}

Awaited results concern the cross-match with the DR1, (Gaia coll., 2016, Arenou et al., 2017) with underlying fundamental questionings as : what are the quasars recognized by the Gaia release and how many objects are concerned? Results will be detailed in the LQAC-4 paper scheduled soon. We give a partial result concerning the ratio of crossmatchs of individual catalogues of the LQAC-4 with respect to he DR1. Notice that 


\begin{tabular}{lllrrrr}
\hline \hline $\begin{array}{l}\text { Catalog } \\
\text { name }\end{array}$ & Flag & $\begin{array}{l}\text { Nature } \\
\text { Radio / } \\
\text { Optical }\end{array}$ & $\begin{array}{r}\text { Nbs of } \\
\text { quasars } \\
\text { in } \\
\text { LQAC-4 }\end{array}$ & $\begin{array}{r}\text { Nb. of } \\
\text { quasars } \\
\text { in }\end{array}$ \\
& & & PR1 & \\
& & & & & & \\
\hline ICRF2 & A & R & 3414 & 2314 & 67.78 \\
VLBA & B & R & 7213 & 4287 & 68.25 \\
VLA & C & R & 1858 & 1228 & 60.71 \\
JVAS & D & R & 2118 & 1373 & 64.82 \\
SDSS & E & 0 & 384834 & 218720 & 56.83 \\
2QZ & F & O & 23660 & 18075 & 76.39 \\
LRG & G & O & 9058 & 2474 & 27.31 \\
FIRST & H & O & 969 & 909 & 93.80 \\
HB & I & O \& R & 6720 & 5991 & 89.15 \\
\hline
\end{tabular}

Table 1. Number of quasars for each catalog of the LQAC-4 compilation with the corresponding number of quasars in common with the Gaia DR1 catalogue

the LQAC-4 contains at all 443725 quasars from which 249071 have a DR1 counterpart, within a 1" search radius. This represents a $56.13 \%$ completness ratio.

\section{Acknowledgements}

This work has made use of data from the European Space Agency (ESA) mission Gaia (https://www. cosmos.esa.int/gaia), processed by the Gaia Data Processing and Analysis Consortium (DPAC, https://www. cosmos.esa.int/web/gaia/dpac/consortium). Funding for the DPAC has been provided by national institutions, in particular the institutions participating in the Gaia Multilateral Agreement.

\section{References}

Arenou, F., Luri, X., Babusiaux, C. et al. 2017, A 8 A 599A, 50A

Becker, R. H., White, R. L., Gregg, M. D. et al. 2001, ApJ. Suppl.135,227

Browne, I. W. A., Patnaik, A. R., Wilkinson, P. N., \& Wrobel, J. M. 1998, MNRAS 293, 257.

Claussen, M. 2006, VLA Calibrator Manual

Croom, S. M., Smith, R. J., Boyle, B. J. et al. 2004, MNRAS 349, 1397

da Angela, J., Shanks, T., Croom, S. M., et al. 2008, MNRAS 383, 565

Fey, A. L., Gordon, D., Jacobs, C. S., et al. 2015, AJ 150, 58.

Gaia Collaboration, Brown, A. G. A., Vallenari, A., Prusti, T. et al. 2016, A $\&$ A595A, 2G

Hewitt, A. \& Burbridge, G. 1993 ApJ.Suppl. 87, No.2, 451

Ma, C., Arias, E. F., Bianco, G. et al. 2009, IERS Technical Note 35

Pâris, I., Petitjean, P., Nicholas, P. R. et al. 2017, A $\&$ A 597, A79

Pier, J. R., Munn, J. A., Hindsley, R. B. et al. 2003, AJ 125, 1559.

Souchay J., Andrei, A. H., Barache, C., et al. 2009, A \& A494, 815.

Souchay, J., Andrei, A. H., Barache, C. et al. 2012, A $\& 3$ A537, A99.

Souchay, J., Andrei, A. H., Barache, C. et al. 2015, A $\&$ A 583, A75.

Véron-Cetty, M. P., \& Véron, P., 1984, A \& A, ESO Scientific Report, No. 1

Véron-Cetty, M. P., \& Véron, P., 2010, A $\&$ A 518, A10

Wilkinson, P. N., Browne, I. W. A., Patnaik, A. R. et al. 1998, MNRAS 300, 790. 